Cite this: Phys. Chem. Chem. Phys., 2014, 16, 17133

Received 7th May 2014, Accepted 20th June 2014

DOI: $10.1039 / c 4 c p 01981 d$

www.rsc.org/pccp

\title{
Energy transfer properties of Rhodobacter sphaeroides chromatophores during adaptation to low light intensity $\dagger$
}

\author{
B. Driscoll, $\neq^{a}$ C. Lunceford $\neq^{a}$ S. Lin, ${ }^{\star b}$ K. Woronowicz, ${ }^{c}$ R. A. Niederman ${ }^{c}$ and \\ N. W. Woodbury*b
}

\begin{abstract}
Time-resolved fluorescence spectroscopy was used to explore the pathway and kinetics of energy transfer in photosynthetic membrane vesicles (chromatophores) isolated from Rhodobacter (Rba.) sphaeroides cells harvested 2, 4, 6 or 24 hours after a transition from growth in high to low level illumination. As previously observed, this light intensity transition initiates the remodeling of the photosynthetic apparatus and an increase in the number of light harvesting 2 (LH2) complexes relative to light harvesting 1 (LH1) and reaction center $(\mathrm{RC})$ complexes. It has generally been thought that the increase in $\mathrm{LH} 2$ complexes served the purpose of increasing the overall energy transmission to the RC. However, fluorescence lifetime measurements and analysis in terms of energy transfer within LH2 and between LH2 and LH1 indicate that, during the remodeling time period measured, only a portion of the additional LH2 generated are well connected to LH1 and the reaction center. The majority of the additional LH2 fluorescence decays with a lifetime comparable to that of free, unconnected LH2 complexes. The presence of large LH2-only domains has been observed by atomic force microscopy in Rba. sphaeroides chromatophores (Bahatyrova et al., Nature, 2004, 430, 1058), providing structural support for the existence of pools of partially connected LH2 complexes. These LH2-only domains represent the light-responsive antenna complement formed after a switch in growth conditions from high to low illumination, while the remaining $\mathrm{LH} 2$ complexes occupy membrane regions containing mixtures of $\mathrm{LH} 2$ and $\mathrm{LH} 1-\mathrm{RC}$ core complexes. The current study utilized a multi-parameter approach to explore the fluorescence spectroscopic properties related to the remodeling process, shedding light on the structure-function relationship of the photosynthetic assembles. Possible reasons for the accumulation of these largely disconnected LH2-only pools are discussed.
\end{abstract}

\section{Introduction}

The photosynthetic apparatus of Rhodobacter (Rba.) sphaeroides is composed of light harvesting complexes 1 and 2 (LH1 and LH2), and the reaction center (RC). In the membrane, the RC and LH1 form a tightly associated core complex (LH1-RC) found mainly in a dimeric form in which each RC is surrounded by an open LH1 ring-like structure, also containing an integral PufX protein. ${ }^{1-3}$ Atomic force microscopy (AFM) studies have shown that the LH1-RC dimers generally align in arrays within the photosynthetic

\footnotetext{
${ }^{a}$ Department of Physics, Arizona State University, Tempe, AZ 85287, USA

${ }^{b}$ Department of Chemistry and Biochemistry and The Biodesign Institute at Arizona State University, Tempe, AZ 85287, USA. E-mail: nwoodbury@asu.edu

${ }^{c}$ Department of Molecular Biology and Biochemistry, Rutgers, The State University of New Jersey, Piscataway, NJ 08854, USA

$\dagger$ Electronic supplementary information (ESI) available. See DOI: 10.1039/ c4cp01981d

\$ These authors contributed equally to this work.
}

membrane and are surrounded by LH2 complexes $^{4}$ (Fig. 1a). The antenna (LH1 and LH2) and the RC protein complexes are thought to assemble in the cytoplasmic membrane, which then invaginates inward, forming chromatophore membrane vesicles that contain the mature apparatus. ${ }^{5-7}$

The structures of the individual LH1, LH2, and RC complexes are known at atomic resolution and their spectroscopic and kinetic properties have been extensively studied (for recent reviews, see ref. 8-10). Each LH1-RC complex in the LH1-RC dimer is composed of 14 pairs of $\alpha / \beta$ subunits and 28 closely associated bacteriochlorophyll $a$ (Bchl $a$ ) molecules surrounding the RC. The LH1 BChl molecules have a $\mathrm{Q}_{\mathrm{Y}}$ absorption band centered at $875 \mathrm{~nm}$ (B875) and a fluorescence spectrum with a maximum at $885 \mathrm{~nm}$. The LH2 complex, in contrast, has two spectroscopically distinct types of BChl molecules. There are 9 pairs of $\alpha / \beta$ subunits in this case containing $9 \mathrm{BChl}$ molecules that are well separated from the others and absorb at $800 \mathrm{~nm}$ (B800) and 18 much more closely associated BChl molecules that absorb at 

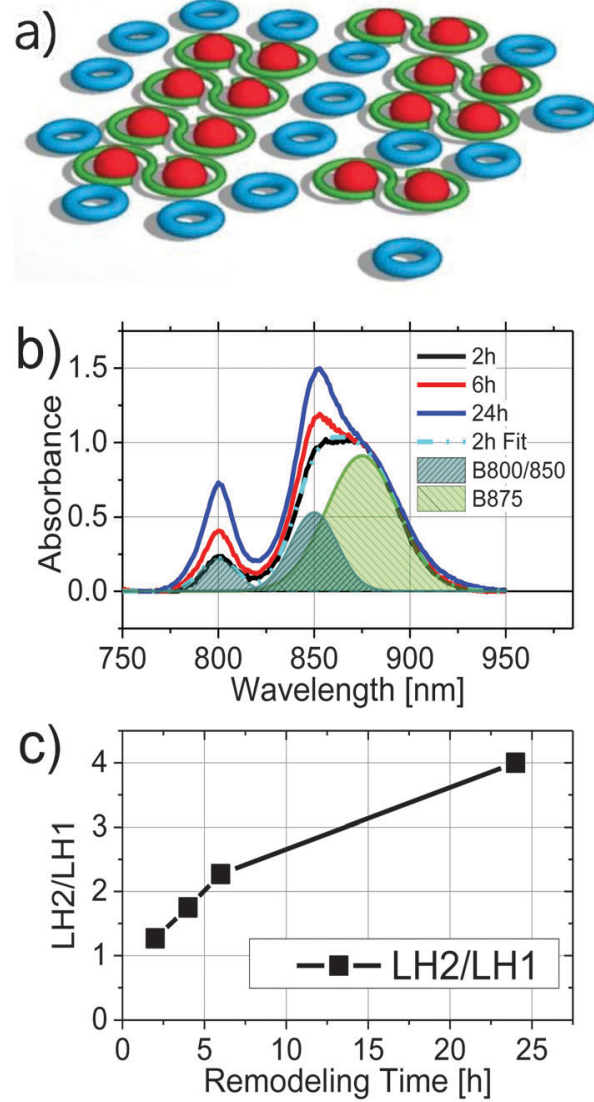

Fig. 1 (a) schematic illustration of photosynthetic units, LH2 (blue), LH1 (green) and RC (red), as arranged in the chromatophore structure; (b) absorption spectra of chromatophores as a function of remodeling time after shifting from highlight to low-light growth conditions, $2 \mathrm{~h}$ (black), $4 \mathrm{~h}$ (red), and $24 \mathrm{~h}$ (blue) normalized to the LH1 absorption maximum at $875 \mathrm{~nm}$. The spectrum at $4 \mathrm{~h}$ remodeling time (not shown) is similar to that of the $6 \mathrm{~h}$ spectrum. The shaded curves are three Gaussians centered at 800,850 , and $875 \mathrm{~nm}$, used in the fitting of the $2 \mathrm{~h}$ absorption spectrum; (c) the relative amplitude ratio of $\mathrm{LH} 2$ and $\mathrm{LH} 1$ at different remodeling times.

$850 \mathrm{~nm}$ (B850). Excitation energy transfer takes place very rapidly from the $\mathrm{B} 800$ ring to the $\mathrm{B} 850$ ring of $\mathrm{LH} 2$ and therefore the fluorescence from this complex is emitted almost entirely by the B850 BChls (fluorescing with a maximum at $860 \mathrm{~nm}$ ). ${ }^{11}$ The fluorescence quantum yield of the RC is much smaller than that from either LH1 or LH2 due to the picosecond quenching of the excited state via electron transfer, ${ }^{12,13}$ and thus there is essentially no observable RC fluorescence from these membranes.

The ratio of LH2 to LH1-RC complexes in mature intracytoplasmic membrane vesicles (chromatophores) depends on both the light intensity and the time the cells have grown at that intensity. When grown under continuous low-light intensity, the LH2/LH1 ratio is larger than when grown under continuous high-light intensity. This is due to an increase in the number of LH2 complexes at low light rather than a change in LH1. When cells are grown anaerobically under high-light and are then shifted to low-light conditions, the photosynthetic apparatus adapts via a process referred to as antenna remodelling. ${ }^{6,14}$ During low-light remodeling, additional LH2 complexes are produced.
The increase in LH2 complexes results in an increase in the total antenna absorption cross-section. In principle, this is consistent with that idea that additional absorbance cross section results in additional energy transfer to the RC, thus partially compensating for the lower light intensity.

If the LH2 that accumulates during low-light adaptation does so to increase the functional absorbance cross section, one might think they would simply add to the existing LH2 pool, increasing its size. However, AFM images of chromatophores showed a heterogeneous distribution of the $\mathrm{LH} 2$ complexes with two types of organization. ${ }^{4}$ Ten to twenty LH2 units are seen to closely associate with the LH1-RC complexes, and this group appears to increase only moderately as remodeling occurs. Most of the accumulation of $\mathrm{LH} 2$ appears to result in the formation of LH2-only domains that are more distal from the LH1-RC arrays. It was suggested that these more distal $\mathrm{LH} 2$ domains may form a light-responsive complement of $\mathrm{LH} 2$ antenna. ${ }^{4}$ A radiolabeling study following the assembly of the photosynthetic apparatus has also shown that mature membranes assemble new LH2 rings during the remodeling process in chromatophores in response to the lowered light intensity. ${ }^{6}$ Once again, it was found that the newly assembled LH2 complexes mainly group in areas not directly associated with existing arrays of LH1-RC complexes and the proximal LH2 complexes that are present under high light. The functionality of these distal LH2 only pools is thus far poorly understood. Recent studies have suggested that this arrangement may slow the turnover rate of the $\mathrm{RC}^{14}$ and/or that under unnaturally high excitation light these extra pigments are involved in photoprotection. ${ }^{15}$

These recent observations suggest that further exploration of the light-harvesting capabilities of the additional pools of LH2 would be enlightening. Here, the energy transfer properties of the proximal LH2 pools are investigated by measuring the picosecond time-resolved fluorescence of photosynthetic membranes at various points during the remodeling process, as well as under various excitation intensities.

\section{Experimental}

\section{Sample preparation}

Whole cells were grown to $\mathrm{OD}_{680}$ of at least 0.2 anaerobically at high-light intensity $\left(1100 \mathrm{~W} \mathrm{~m}^{-2}\right)$ and then switched to a lower intensity $\left(100 \mathrm{~W} \mathrm{~m}^{-2}\right)$, initiating the remodeling process. The cells collected at 2, 4, 6, and 24 hours after initiation of low light conditions were then passed through a French press and chromatophores were isolated from the lysate using rate-zone ultracentrifugation. ${ }^{14}$ Absorption spectra of these samples were obtained using a Varian UV-visible spectrophotometer (Cary 5, Varian).

\section{Time-resolved fluorescence spectroscopy}

Fluorescence emission spectra were recorded as a function of delay time using a streak camera system described previously. ${ }^{16}$ The excitation pulse was generated from a Nd:YVO4-pumped, model-locked titanium:sapphire laser producing 100 fs pulses 
at a repetition rate of $76 \mathrm{MHz}$ and a wavelength of $800 \mathrm{~nm}$ (Coherent Inc., Verdi-18 and Mira-900). The excitation intensity was controlled using neutral density filters, and was kept at $1 \mathrm{~mW}\left(5.3 \times 10^{7}\right.$ photons per pulse or $7.6 \times 10^{8}$ photons per pulse per $\mathrm{cm}^{2}$ ) for the study of fluorescence kinetics as a function of remodeling time, but was varied between $0.1 \mathrm{~mW}$ and $20 \mathrm{~mW}$ for the excitation intensity dependence study. A beam diameter of $3 \mathrm{~mm}$ was set to excite the maximal volume of the sample in the $3 \times 3 \mathrm{~mm}^{2}$ cuvette while minimizing artefacts resulting from scattering. A long-pass filter, with 50\% transmittance at $825 \mathrm{~nm}$ was placed between the sample and the detector to reduce scattered excitation light, and an IR sheet polarizer was set to $54.7^{\circ}$ to collect fluorescence at the "magic angle" relative to the excitation polarization. The fluorescence was then focused onto the entrance slit of a spectrograph (Chomex 250IS) coupled to a streak camera and a CCD (Hamamatsu C5680). Individual fluorescence measurements involved integration times between 1 and $6 \mathrm{~h}$, depending on the sample. Time-resolved fluorescence spectra were recorded over a wavelength range of 830 to $940 \mathrm{~nm}$, and an $800 \mathrm{ps}$ time window. The full-width-at-halfmaximum of the instrument response function was $\sim 10$ ps.

\section{Data analysis and modeling}

Time resolved fluorescence emission spectra as a function of decay time were corrected for the wavelength sensitivity of the detecting system, and globally fit to a sum of exponential decays (eqn (1)) using a locally written software package, ASUFIT. The quality of the fit was judged by the reduced chi squared value. Fitting was performed with the least number of exponential decay components statistically required and all fits yielded reduced chi squared values $\leq 1.7$ (additional components did not reduce the chi squared value further).

$$
F(\lambda, t)=\sum_{i} \operatorname{DAS}_{i}(\lambda) \mathrm{e}^{-t / \tau_{i}}
$$

In eqn (1), $\operatorname{DAS}_{i}(\lambda)$ is the wavelength dependent amplitude of the $i$ th decay component (Decay Associated Spectra), and $\tau_{i}$ is the $i$ th exponential time constant.

\section{Results}

\section{Absorption spectroscopic properties of chromatophores as a function of remodeling time}

Fig. 1b compares the absorption spectra of 2,6 , and $24 \mathrm{~h}$ samples normalized at $875 \mathrm{~nm}$, the wavelength of LH1 absorption maximum. The rise in the B850 peak was only slightly greater in the $6 \mathrm{~h}$ relative to $4 \mathrm{~h}$ chromatophores $(4 \mathrm{~h}$ spectrum not shown). It is clear that as the remodeling proceeded, relative intensities of the $\mathrm{LH} 2$ absorption bands at $800 \mathrm{~nm}$ and $850 \mathrm{~nm}$ increased. In order to determine the amount of LH2 relative to LH1 as a function of the remodeling process, each absorption spectrum was fit by a sum of three Gaussians centered at 800,850 , and $875 \mathrm{~nm}$, corresponding to absorption in LH2 (B800 and B850) and LH1 (B875), respectively. The three Gaussian bands obtained from fitting of the $2 \mathrm{~h}$ sample are illustrated in Fig. $1 \mathrm{~b}$ (shaded areas). The ratio of the amplitudes at $800 \mathrm{~nm}$ and $875 \mathrm{~nm}$ is plotted as a function of remodeling time and shown in Fig. 1c. Consistent with prior studies, ${ }^{14}$ the amount of LH2 increased relative to LH1 with remodeling time, with the $24 \mathrm{~h}$ sample showing $\sim 3$ fold more LH2 than the $2 \mathrm{~h}$ sample.

\section{Fluorescence kinetics of LH2 and LH1 from chromatophores at different stages of remodeling}

To explore energy transfer in chromatophores as a function of the remodeling process, time-resolved fluorescence spectra from LH2 and LH1 were analyzed kinetically following selective excitation of the higher energy band of LH2 (B800) at $800 \mathrm{~nm}$. The optical density of each sample was adjusted to 0.2 at the excitation wavelength to ensure that the same number of photons were absorbed by each at a given excitation intensity.

All the experiments were performed at room temperature and samples were not stirred during data collection. Under the conditions described above, the reaction centers were in the closed state during the measurements (i.e., the majority of the reaction centers in the sample had the primary electron donor, $\mathrm{P}$, oxidized). Global fitting of the fluorescence decays to a series of exponential decay terms (see Methods) required three components for an adequate fit. The resulting DAS for each remodeling time sample are shown in Fig. 2, and the decay lifetimes as a function of remodeling time are plotted in Fig. 3. Because the excitation energy transfer from the $800 \mathrm{~nm}$ to the $850 \mathrm{~nm}$ absorbing BChls of LH2 takes place on the subpicosecond time scale, the fluorescence signal from the $800 \mathrm{~nm}$ BChls of LH2 is very weak and no attempt was made to measure it or to include that process in the kinetic analysis. The amplitude spectrum of the first kinetic component (DAS 1 , black curves in Fig. 2), has a positive band peaking at $859 \mathrm{~nm}$ (LH2) and a negative band at $885 \mathrm{~nm}$ (LH1) at all times during remodeling, consistent with energy transfer from LH2 to LH1. This kinetic component has been previously observed in mature photosynthetic membranes. ${ }^{8}$ The time constant associated with this process is between 20-40 ps as shown in Fig. 3(a). The amplitude spectrum of the second component (DAS ${ }_{2}$, red curves in Fig. 2) is again largely similar at all times during remodeling and has a positive band centered at $880 \mathrm{~nm}$, which corresponds primarily to fluorescence from LH1. The decay time constant associated with this component is consistent with fluorescence kinetics previously recorded for LH1-RC complexes; ${ }^{17}$ it rises rapidly from $\sim 70$ to $\sim 130 \mathrm{ps}$ after the $2 \mathrm{~h}$ time point and then remains nearly constant (Fig. 3b). The amplitude spectrum of the third component, $\mathrm{DAS}_{3}$ (blue curves in Fig. 2), is blue-shifted relative to $\mathrm{DAS}_{2}$, and the extent of the blue shift increases with the remodeling time. Furthermore, a nearly 6-fold increase in the lifetime of this component, from 170 to $970 \mathrm{ps}$, is observed as remodeling progresses (Fig. 3c). The 970 ps time constant seen at $24 \mathrm{~h}$ for $\mathrm{DAS}_{3}$ is similar to the longest-lived lifetime seen for chromatophores made from an LH2-only mutant ( $\sim 1.2 \mathrm{~ns}),{ }^{18}$ suggesting that the $\mathrm{LH} 2$ giving rise to this component does not undergo efficient energy transfer to LH1 (note that at the $2 \mathrm{~h}$ time point, the 20, 70 and 190 ps components are probably mixed in the fitting, a possibility supported by the observed 

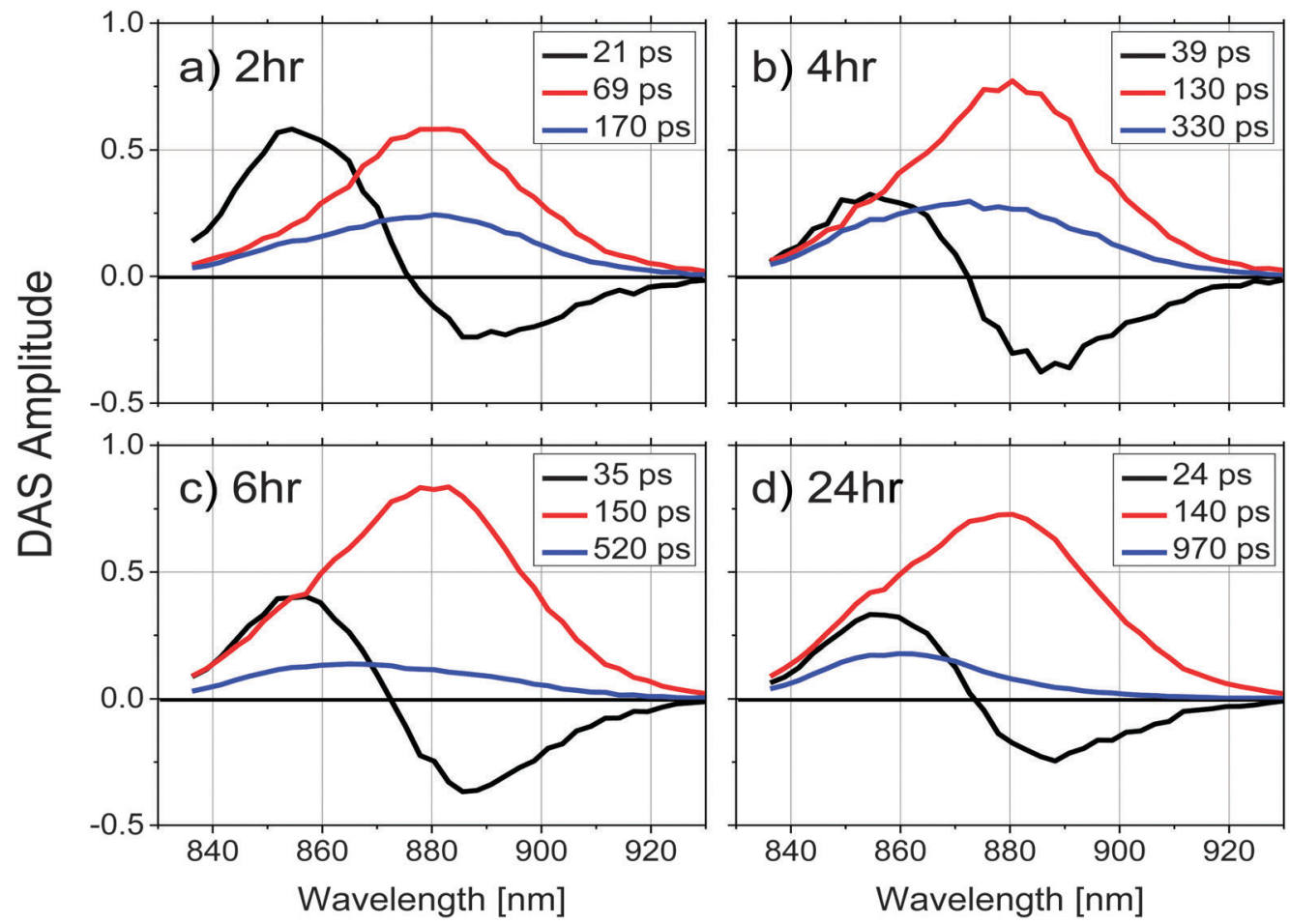

Fig. 2 Decay associated spectra (DAS) for chromatophores after a remodeling time of (a) 2, (b) 4, (c) 6 and (d) 24 hours, obtained from a 3-exponential global fitting of the time-resolved fluorescence emission spectra. Samples were adjusted to have the same absorbance at $800 \mathrm{~nm}$, the laser excitation wavelength. A 3D plot of a data set with residuals (Fig. S3, ESI $\dagger$ ) illustrates the fitting quality.

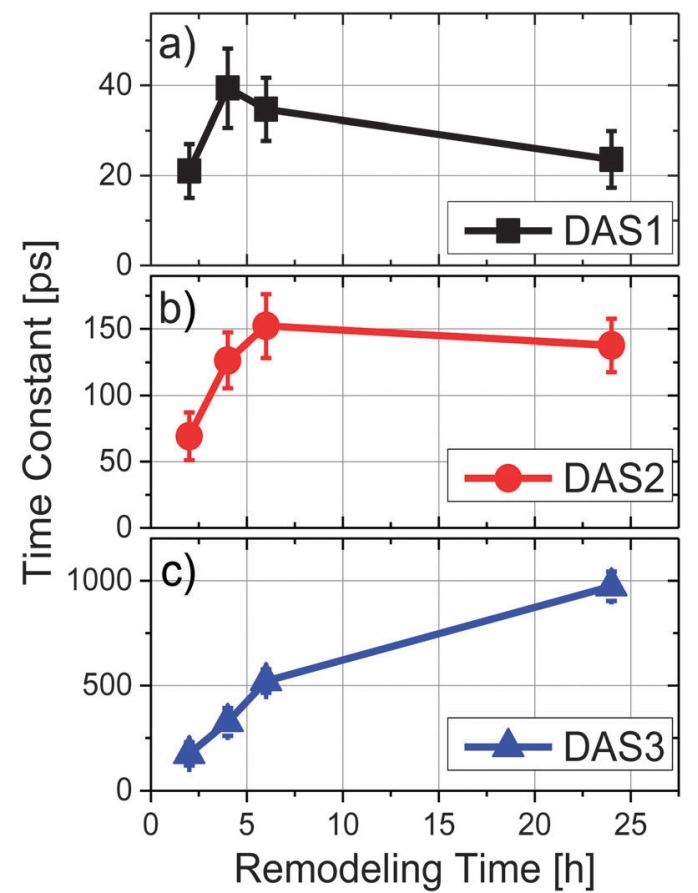

Fig. 3 Time constants as a function of remodeling time where (a) DAS1, (b) DAS2 and (c) DAS3 correspond to the short (20-30 ps), middle (70-150 ps) and long (170 ps-1 ns) terms, respectively.

gradual blue shift in the DAS spectrum of the slow component at longer remodeling times). This likely explains the apparent $\sim 2$-fold increase in lifetime of the first and second components between 2 and $4 \mathrm{~h}$.

To evaluate the contributions of LH1 and LH2 to each DAS independently, the three amplitude spectra associated with each sample were analyzed by fitting the wavelength region between $830 \mathrm{~nm}$ and $930 \mathrm{~nm}$ of each spectrum to 2 Gaussian functions with peaks centered at 860 and $890 \mathrm{~nm}$. Both the peak wavelength and the width of each Gaussian were held constant during the fitting, allowing only the amplitudes of the two spectral components to vary. An example of such a spectral decomposition is illustrated in Fig. 4d. The DAS of different samples were normalized to the initial fluorescence intensity recorded close to time zero. The relative amplitudes of the resulting fits are shown in Fig. $4 \mathrm{a}$ and $\mathrm{b}$ for $\mathrm{DAS}_{1}$ and $\mathrm{DAS}_{3}$ respectively, and a ratio of the peak amplitudes of the Gaussian functions centered at $860 \mathrm{~nm}$ and $890 \mathrm{~nm}$ (corresponding to LH2 and LH1, respectively) as a function of remodeling time are given for the longest-lived component $\left(\mathrm{DAS}_{3}\right)$ in Fig. 4c. Because $\mathrm{DAS}_{2}$ does not significantly change spectrally during remodeling, it is not shown.

The relative contributions of LH2 and LH1 in each DAS illustrated in Fig. 4a-c show that: (1) when LH2 is excited, excitation energy is transferred to LH1 with a time constant of $\sim 40 \mathrm{ps}$, (2) the LH1 excited state formed decays with a time constant of $\sim 150 \mathrm{ps}$, and (3) the longest decay process, which takes place on the order of hundreds of ps, shows the most pronounced change as a function of antenna remodeling time and is mainly due to fluorescence from $\mathrm{LH} 2$. The DAS associated 

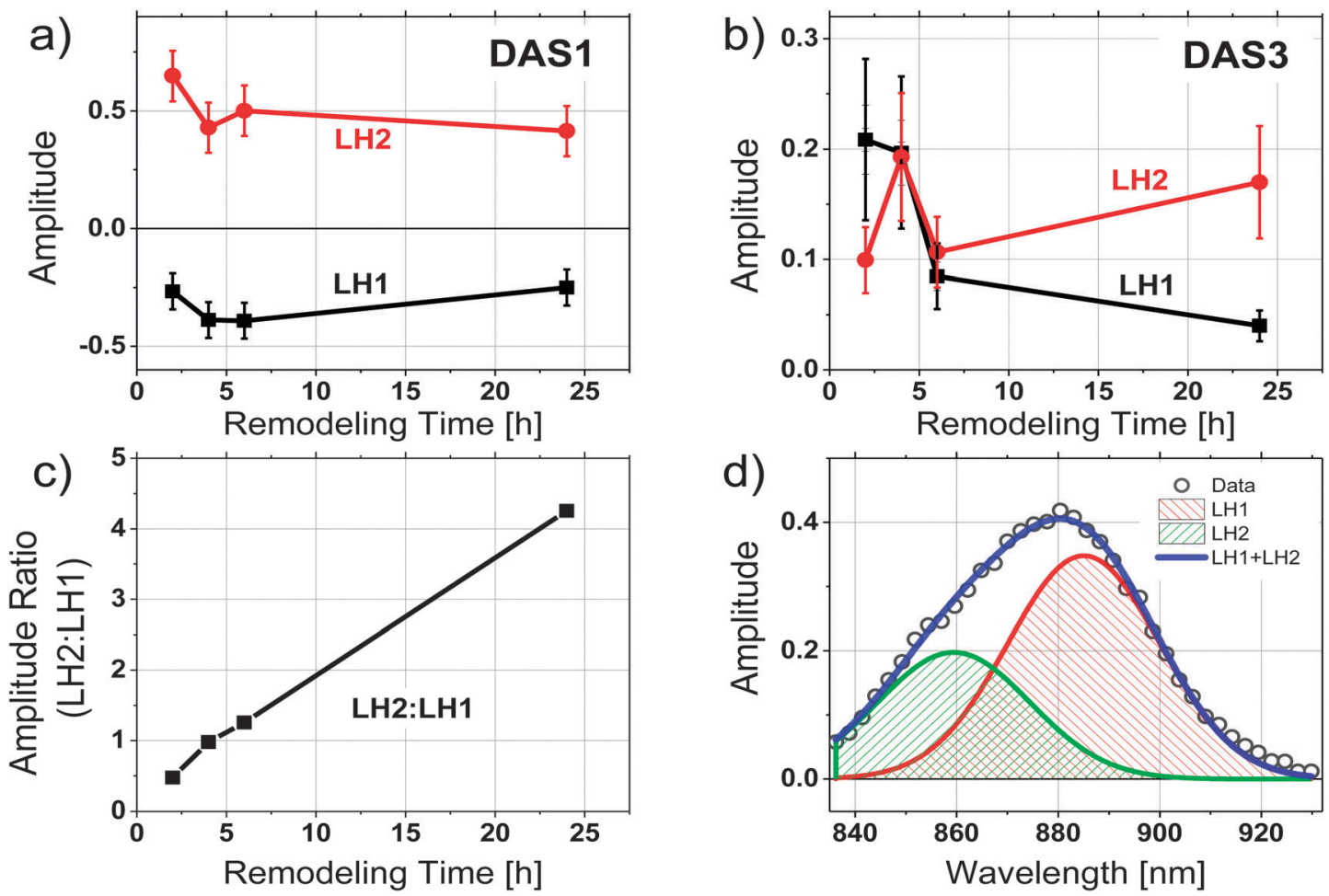

Fig. 4 (a) Amplitudes of Gaussian curves used to characterize the trends of fluorescence contributions from LH2 and LH1 as a function of remodeling time. (a) The short time component $\left(D^{\prime} S_{1}\right)$. Amplitudes of the LH1 wavelengths ( $\sim 885 \mathrm{~nm}$ ) are negative as a result of the DAS fitting, which indicates a rise in fluorescence from LH1, (b) identical analysis on the long-lived time component (DAS $)_{3}$, (c) ratio of the amplitudes LH2: LH1 (860 $\mathrm{nm}: 885 \mathrm{~nm}$ ). (d) Illustration of spectral deconvolution into two Gaussians of $\mathrm{DAS}_{3}$ from the $2 \mathrm{~h}$ sample at $1 \mathrm{~mW}$ excitation intensity.

with this decay also indicates an increased fluorescence contribution from LH2 as remodeling proceeds (it shifts to the blue, Fig. 2). These results are consistent with the idea that as remodeling progresses, there is a larger and larger amount of $\mathrm{LH} 2$ generated that is not well connected to the remainder of the photosynthetic apparatus in terms of excitation energy transfer. Apparently, there are at least two pools of LH2 complexes present with distinct kinetic profiles.

\section{Excitation intensity dependence study}

In order to further explore the origins of the different fluorescence components, the excitation intensity dependence of the time resolved fluorescence spectra was measured as a function of remodeling time for the 2 and $24 \mathrm{~h}$ samples. Excitation intensities ranging from $0.1-20 \mathrm{~mW}$ were used, (note, $1 \mathrm{~mW}=7.6 \times 10^{8}$ photons per pulse per $\mathrm{cm}^{2}$ at the excitation wavelength) and both global fitting and Gaussian deconvolution of the resulting amplitude spectra were performed, as described above.

Similar to the results described above, 3 kinetic components with lifetimes in the range of $20-30 \mathrm{ps}, 100-150 \mathrm{ps}$, and 600-1000 ps were resolved from each data set. The spectral profile of the middle component, dominated by the decay of LH1, remains relatively unchanged under all excitation intensities for both the $2 \mathrm{~h}$ and $24 \mathrm{~h}$ samples. Significant spectral changes in the DAS as a function of excitation intensity were observed in DAS1 and DAS3 (Fig. S1 and $\mathrm{S} 2, \mathrm{ESI} \dagger$ ). Two Gaussian functions, representing the fluorescence from LH2 and LH1, were used to fit the DAS1 and DAS3 of the
2 and 24 h samples at each excitation intensity. Fig. 5 compares the amplitudes of the resulting two Gaussian functions plotted as a function of excitation intensity, after normalizing to the amplitude of DAS2. The positive amplitude of DAS1, representing the maximum of LH2 emission at $860 \mathrm{~nm}$, increases with the excitation intensity (Fig. 5a and c), while the negative amplitude at $890 \mathrm{~nm}$ (LH1) reduces with the excitation intensity. Because of the nature of the spectrum associated with energy transfer between LH2 and LH1, it is not clear whether the observed changes represent an increase in contribution from $\mathrm{LH} 2$, a decrease in contribution from LH1, or both. DAS3, in contrast, shows a decrease in the relative contribution from $\mathrm{LH} 2$ for the $24 \mathrm{~h}$ sample and a decrease in both LH2 and LH1 for the 2 h sample (Fig. $5 \mathrm{~b}$ and $\mathrm{d}$ ). These results are most easily explained in terms of annihilation that occurs in both LH1 and LH2 at the $2 \mathrm{~h}$ time point and primarily takes place in $\mathrm{LH} 2$ at the $24 \mathrm{~h}$ time point.

\section{Discussion}

Previous work has shown that when cell growth conditions are shifted from high to low light, the amount of LH2 in the chromatophores increases. Logically, one would assume this larger absorbance cross section per reaction center would result in increased light harvesting at low light. Structural studies of the remodeling process have begun to provide a picture of the evolving organization of the photosynthetic apparatus as 

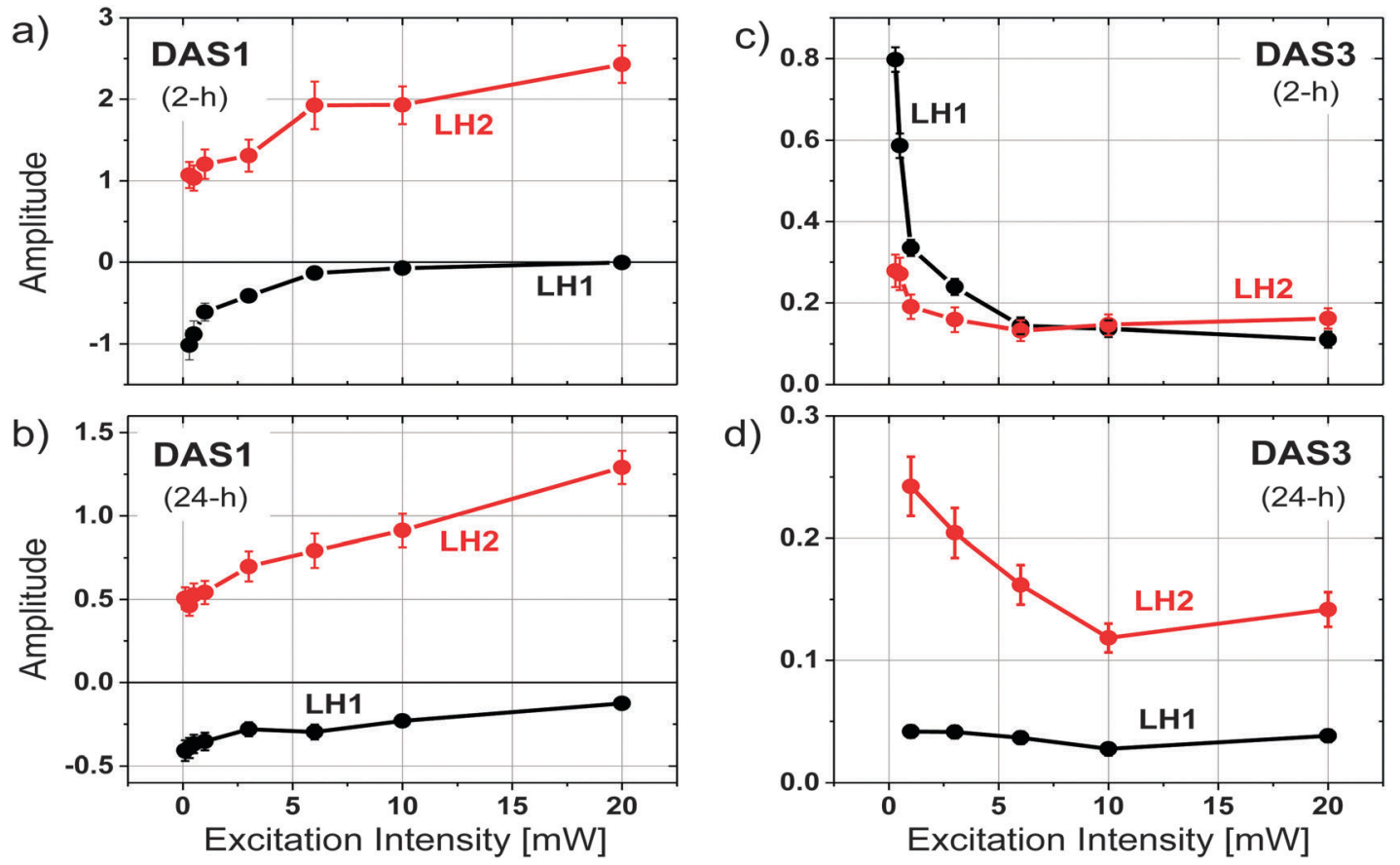

Fig. 5 Amplitudes of Gaussian bands used to characterize the trends of fluorescence contributions from LH2 and LH1 as a function of excitation intensity. (a) The short time component (DAS1) of the $2 \mathrm{~h}$ sample. (b) DAS1 for the $24 \mathrm{~h}$ sample. (c) The long-time component (DAS3) of the $2 \mathrm{~h}$ sample. (d) DAS3 for the $24 \mathrm{~h}$ sample as a function of excitation intensity.

additional LH2 is added. ${ }^{6}$ The observation of two types of organization for LH2 complexes, one type of LH2 closely associated with the linear arrays of dimeric LH1-RC complexes and the other type located outside these arrays in larger clusters, raises questions regarding how this architectural design is related to light-harvesting function. The goal of the current study is to explore the functional evolution of the light harvesting apparatus during the remodeling transition, with particular attention paid to the function of LH2 as an extended antenna.

\section{Changes in the distribution of excited states between LH1 and LH2 pools during remodeling}

The most significant result from the above analysis is that the increase in LH2 with remodeling does not correspond to an increase in energy transfer from the LH2 to LH1-RC complexes. The additional LH2 complexes assembled under low-light conditions seem to form LH2 pools, which are poorly connected or completely disconnected from the LH1-RC arrays. A qualitative scheme depicting possible organizational changes as LH2 complexes assemble in the remodeled photosynthetic unit is shown in Fig. 6. At early remodeling times, an array of core complexes is surrounded by a small number of LH2 complexes, as has been suggested in previous studies. ${ }^{4,6}$ As remodeling of the photosynthetic apparatus proceeds, more LH2 complexes are added but at least a portion of these newly-added LH2 complexes are functionally disconnected or poorly connected to the LH1-RC dominated domains. This results in a low efficiency of excitation energy transfer from the distal pool, and an increasingly long decay time approaching the inherent decay time of isolated LH2 complexes.

Compelling evidence for this scheme is seen in Fig. 4c, where the ratios of contributions from LH2 and LH1 to the longest-lived amplitude spectrum, DAS3, is given. The plot indicates a significant increase in fluorescence contribution from LH2 relative to LH1. This is of particular interest when combined with the observation that as remodeling time increases, the lifetime of DAS3 approaches the lifetime of isolated LH2, $1.2 \mathrm{~ns}$ (see Fig. 3c). This suggests that the increase in LH2 between 2 and $24 \mathrm{~h}$ results in a population of LH2 complexes that are poorly connected to LH1 complexes, and thus RCs, at least at the level of excitation intensity used in the measurement. The kinetic evidence presented here for the existence of disconnected, or poorly connected, LH2 pools matches very well with the AFM observations of LH2-only clusters formed during growth under low-light. ${ }^{4}$

\section{Different mechanisms of the two types of LH2 pools in handling excess excitation}

Exciton redistribution between LH2 and LH1 complexes due to the accumulation of LH2 during remodeling were further explored by measuring the excitation intensity dependence of the time resolved fluorescence. Interestingly, the DAS3 of the $2 \mathrm{~h}$ sample additionally exhibits a decrease in LH1 fluorescence, providing evidence for annihilation in LH1 (Fig. 5c and Fig. S1c, ESI $\dagger$ ). The similar trend of the excitation intensity dependent decrease in the fluorescence of the LH1 and LH2 components of the longer-lived fluorescence (Fig. 5b) indicates that there exists a strong coupling between LH2 and LH1 


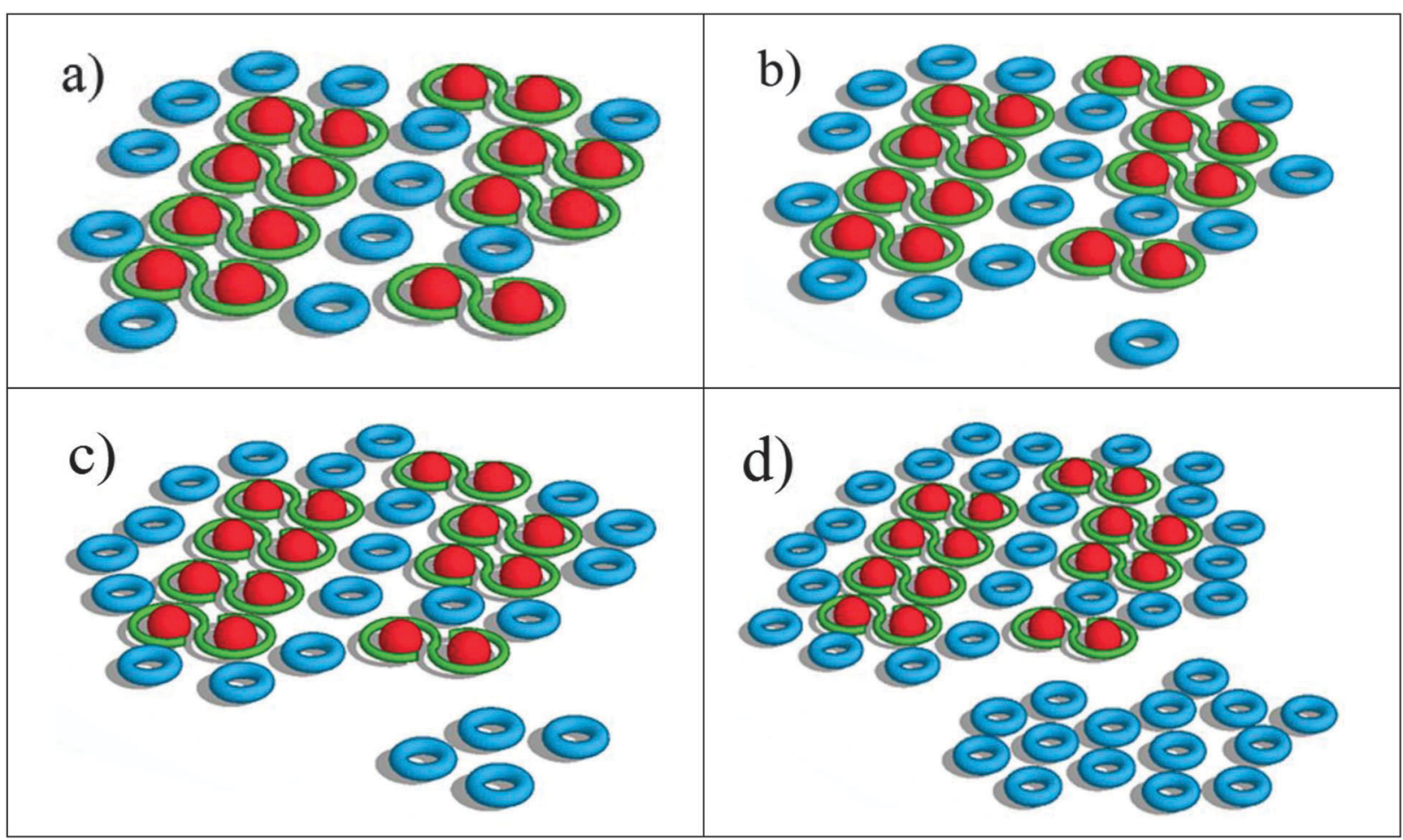

Fig. 6 Schematic of LH2 accumulation as remodeling time increases. Representations are of (a) $2 \mathrm{~h}$, (b) $4 \mathrm{~h}$, (c) $6 \mathrm{~h}$ and (d) $24 \mathrm{~h}$. The ratio of LH2 : LH1-RC, as well as the ratio of the two different pools of $\mathrm{LH} 2$ complexes matches that used in the simulation.

complexes in the $2 \mathrm{~h}$ chromatophores. In contrast, the kinetic behavior of the $24 \mathrm{~h}$ sample shows a decrease in the part of the long DAS3 component attributed to LH2 as the excitation intensity is increased, while the LH1 portion of DAS3 remains largely unchanged (Fig. 5d). This indicates that in the $24 \mathrm{~h}$ sample, excitation annihilation occurs predominantly in the LH2 only pools, again, consistent with the proposed notion of functionally disconnected pools of LH2.

\section{Kinetic simulation}

The observed fluorescence kinetics as a function of remodeling time were reproduced using a simple model for the physical relationship between the photosynthetic constituents and were found to be consistent with the above interpretation. In order to put the scheme in Fig. 6 on a more quantitative footing, the system was simulated in terms of a random walk energy transfer network. For these simulations, all reaction centers were assumed to be closed (note that closed reaction centers still quench excitations from LH1). Each exciton carried a probability to hop between different LH2 complexes with a rate constant of $100 \mathrm{~ns}^{-1}$, corresponding roughly to the published time constant for energy transfer between LH2 complexes. ${ }^{8}$ Each LH2 and LH1 complex is considered as an individual point on a $2 \mathrm{D}$ grid. The model allowed for radiative decay (fluorescence) and singlet-singlet excited state annihilation within both LH2 and LH1 pools. Annihilation was assumed to occur whenever two excitons met in a single complex, and fluorescence was assumed to occur with rate constants of $0.83 \mathrm{~ns}^{-1}$ and $10 \mathrm{~ns}^{-1}$ for LH2 and LH1, respectively, which are the fluorescence decay rates from LH2-only and LH1-only mutants. ${ }^{8}$ At any boundary between $\mathrm{LH} 2$ and $\mathrm{LH} 1$, the probability of transfer from LH2 $\rightarrow$ LH1 was set at 1/10, and back transfer from LH1 $\rightarrow$ LH2 at $1 / 50$. These values were estimated from previously reported characteristic times of energy transfer and the ratio verified by Boltzmann energy statistics. In the 4, 6, and $24 \mathrm{~h}$ simulations, a portion of the LH2 complexes were assumed to reside in LH2-only pools that were not as well connected to LH1 as other LH2, which was done by reducing the transfer rate into and out of the pools from $100 \mathrm{~ns}^{-1}$ to $0.67 \mathrm{~ns}^{-1}$. Additionally, the ratio of LH2/LH1 used in the simulations was obtained from the ratios calculated from the absorption spectra as given in Fig. 1b. These ratios were then combined with known extinction coefficients to reveal relative concentrations of LH2 and LH1.

Output from the simulation provided fluorescence counts as a function of time (one time interval corresponded to $1 \mathrm{ps}$ ). Attempts to fit this output globally, as was done with the experiment data, proved unsuccessful presumably due to the bimodal nature of the output (LH1 fluorescence and LH2 fluorescence). Time constants associated with each kinetic process were thus obtained by individually fitting each fluorescence $v s$. time data set with a minimum set of exponential decay components. Fits readily provided time constants consistent with the experimental results; offering a fast component of $\sim 30 \mathrm{ps}$, seen as a decay in the LH2 fluorescence and a rise in LH1, a component of 100-150 ps, dominated by LH1 fluorescence, and a long-lived component dominated by LH2 which ranged from $170-200$ ps before the inclusion of LH2 only pools and 200-1000 ps after. 

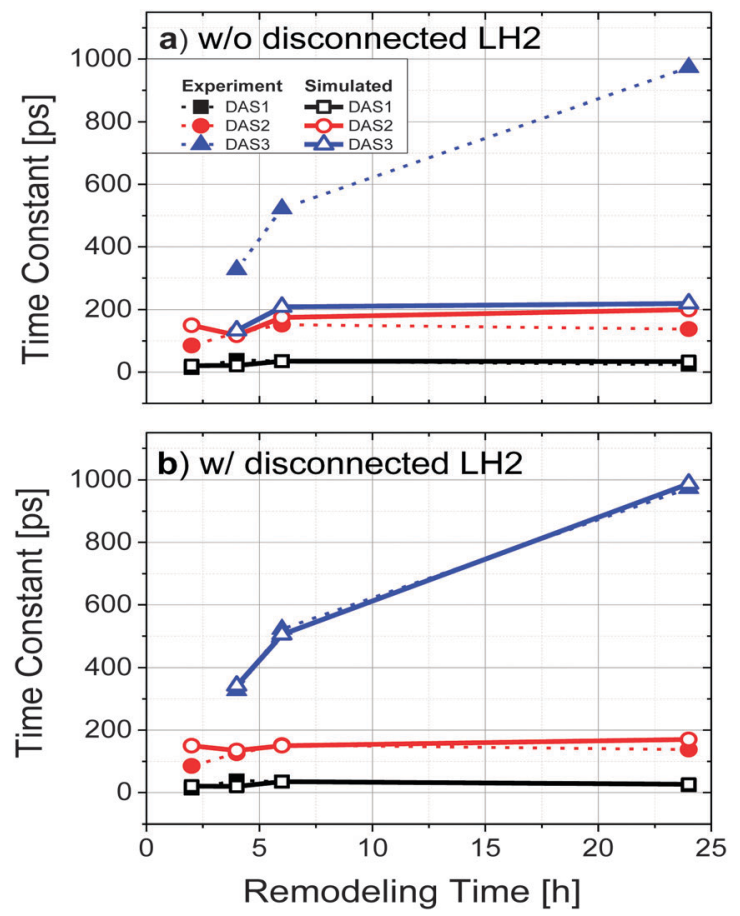

Fig. 7 Comparison of simulation (open symbols with solid lines) and streak camera derived long-lived time-constants (DAS3, solid symbols with dashed lines) (a) without and (b) with a functionally disconnected pool for samples at different remodeling times under low excitation intensity.

The results of these simulations are given in Fig. 7. Fig. 7a shows the simulation as a function of remodeling time without the addition of a functionally weakly connected or disconnected pool of LH2. Using the literature-value rate constants given above and assuming homogeneity in rate constants across like constituents, it was not possible to obtain simulation data approximating the long component time constant $\left(\mathrm{DAS}_{3}\right)$.

Instead, much faster decay time constants were obtained. Inclusion of a portion of $\mathrm{LH} 2$ in a disconnected pool, as described above, yielded results (Fig. 7b) that were much more consistent with the data seen in Fig. 3, supporting the concept that much of the added LH2 absorbance is due to unconnected or poorly connected LH2 complexes.

\section{Possible functions of the additional LH2 pools formed during the remodeling}

Previous studies have shown an increasing amount of LH2 during remodeling, when cell growth shifted from high to low light intensity. ${ }^{6,14}$ This expansion of $\mathrm{LH} 2$ pools would be expected to enhance the light-harvesting capability of the antenna network to maintain photosynthetic activity in the system. However, the studies described above imply that the added LH2 is largely unconnected to the RC, a concept also supported by previous radiolabelling studies. ${ }^{6}$

The role of light level in modulating the connectivity of energy transfer in antennas in vitro has been studied in the past, ${ }^{15}$ suggesting that there may be an inherent response even in the absence of changes in the number of LH2 complexes. The in vivo remodeling process has been observed in other species of purple bacteria and it has been suggested that it may play a role in photoprotection ${ }^{19}$ or in modulating the rate at which reaction centers turnover, ${ }^{14,20}$ but the fundamental question of why it would be advantageous to generate large amounts of unconnected LH2 upon decreasing the light level remains unanswered.

In considering this question, it is important to remember that laboratory growth conditions are quite different from those experienced in the organism's natural habitat. The "high" level of light used for bacterial growth in these studies is in the range of the maximum solar intensity level that would hit the surface of the earth. The "low" level is one tenth of that. In the natural environment, diurnal light-dark cycles and clouds as well as the level of shading from surrounding bacteria or other organisms in a liquid environment, mean that the dynamic range of light exposure any bacterium sees may be much greater than ten-fold (and probably shifted significantly downward from the intensities used here). Furthermore, the duration and frequency of exposure to high and low light levels is likely very different in the natural environment compared to the laboratory. This makes it difficult to extrapolate the current results to natural function. What one can say is that decreasing the average light intensity seen by the bacterium over hours initiates the production of LH2 complexes that are not all initially involved in functional light harvesting. It is possible, for example, that production of $\mathrm{LH} 2$ and its functional incorporation into the photosynthetic apparatus are controlled by separate environmental factors that are not appropriately mimicked in the growth regime used in this study.

\section{Acknowledgements}

This work was supported by National Science Foundation award No. PHY-1057827 (NWW, SL, BD, and CL) and subaward No. 12-764 (RAN), and by U. S. Department of Energy (DE-FG0208ER15957) from the Chemical Sciences, Geosciences and Biosciences Division, Office of Basic Energy Sciences, Office of Science (RAN). We thank Joseph M. Kay and Arpit A. Patel for assistance in membrane preparation, and Dr A. Freiberg for helpful discussions.

\section{Notes and references}

1 T. Walz, S. J. Jamieson, C. M. Bowers, P. A. Bullough and C. N. Hunter, J. Mol. Biol., 1998, 282, 833-845.

2 R. Niederman, Structure, function and formation of bacterial intracytoplasmic membranes, Springer-Verlag, Heidelberg, Berlin, 2006.

3 R. E. Blankenship, Molecular Mechanisms of Photosynthesis, Iowa State University Press, Blackwell Science Ltd., Ames, Iowa, 2002.

4 S. Bahatyrova, R. N. Frese, C. A. Siebert, J. D. Olsen, K. O. van der Werf, R. van Grondelle, R. A. Niederman, P. A. Bullough, C. Otto and C. N. Hunter, Nature, 2004, 430, 1058-1062.

5 R. A. Niederman, D. E. Mallon and J. J. Langan, Biochim. Biophys. Acta, 1976, 440, 429-447. 
6 C. N. Hunter, J. D. Tucker and R. A. Niederman, Photochem. Photobiol. Sci., 2005, 4, 1023-1027.

7 M. Koblizek, J. D. Shih, S. I. Breitbart, E. C. Ratcliffe, Z. S. Kolber, C. N. Hunter and R. A. Niederman, Biochim. Biophys. Acta, 2005, 1706, 220-231.

8 R. J. Cogdell, A. Gall and J. Kohler, Q. Rev. Biophys., 2006, 39, 227-324.

9 C. L. Tavano and T. J. Donohue, Curr. Opin. Microbiol., 2006, 9, 625-631.

10 J. N. Sturgis and R. A. Niederman, Photosynth. Res., 2008, 95, 269-278.

11 V. Sundstrom, T. Pullerits and R. van Grondelle, J. Phys. Chem. B, 1999, 103, 2327-2346.

12 N. W. Woodbury and J. P. Allen, in Anoxygenic Photosynthetic Bacteria, ed. R. E. Blankenship, M. T. Madigan and C. E. Bauer, Kluwer Academic Publishers, Dordrecht, 1995, vol. 2, pp. 527-557.
13 W. Zinth and J. Wachtveitl, ChemPhysChem, 2005, 6, 871-880.

14 K. Woronowicz, D. Sha, R. N. Frese and R. A. Niederman, Biochemistry, 2011, 50, 4819-4829.

15 G. J. Magis, M. J. den Hollander, W. G. Onderwaater, J. D. Olsen, C. N. Hunter, T. J. Aartsma and R. N. Frese, Biochim. Biophys. Acta, 2010, 1798, 637-645.

16 B. Thangaraj, C. C. Jolley, I. Sarrou, J. B. Bultema, J. Greyslak, J. P. Whitelegge, S. Lin, R. Kouril, R. Subramanyam, E. J. Boekema and P. Fromme, Biophys. J., 2011, 100, 135-143.

17 A. Freiberg, J. P. Allen, J. C. Williams and N. W. Woodbury, Photosynth. Res., 1996, 48, 309-319.

18 H. Bergstrom, V. Sundstrom, R. Vangrondelle, T. Gillbro and R. Cogdell, Biochim. Biophys. Acta, 1988, 936, 90-98.

19 S. Scheuring and J. N. Sturgis, Science, 2005, 309, 484-487.

20 K. Woronowicz and R. A. Niederman, Adv. Exp. Med. Biol., 2010, 675, 161-178. 